\title{
Dal senso alla ragione, dalla ragione al senso
}

Aniello Montano, "Methodos". Aspetti dei metodi e dei processi cognitivi nella Grecia Antica, Napoli 2014.

ENRICO VOLPE / University of Salerno /

Il libro che qui presento è una raccolta di saggi (molti dei quali già editi) di Aniello Montano, dal titolo Methodos. Aspetti dei metodi e dei processi cognitivi nella Grecia Antica. Si tratta di un testo che fornisce un'analisi critica ampia ed al tempo stesso esaustiva principalmente del tema della gnoseologia nella filosofia greca antica. Il merito maggiore dell'opera è non solo quello di avere riportato alla luce una tematica sempre attuale, $\mathrm{ma}$ anche e soprattutto quello di avere articolato l'opera restituendo l'immagine di un vero e proprio dibattito redivivo.

Uno degli aspetti centrali su cui l'autore pone la sua attenzione è quello inerente il rapporto che intercorre tra ragione e mondo fenomenico. Il primo saggio preso in esame è quello concernente il pensiero di Eraclito (Mathesis e noos in Eraclito, pp. 15-24). Montano esordisce qui con una domanda concreta «Eraclito difensore del valore conoscitivo delle testimonianze sensibili e teorico dell'induzione oppure Eraclito rappresentante del razionalismo gnoseologico?» La provocazione iniziale dell'autore sembra già mostrare come la filosofia dell'Oscuro di Efeso sia in controtendenza con queste affermazioni dicotomiche; infatti Eraclito sembrerebbe porre ragione e senso come due elementi in simbiosi e non in contraddizione tra loro. Ciò è più chiaro attraverso la fondamentale 
distinzione tra mathesis e noos. La prima viene intesa come un «"apprendimento" derivante dalla collaborazione di tutti i sensi» (p. 18), mentre il secondo denota propriamente l'attività intellettuale. Il porre in essere questo dualismo è però un'attività erronea, prerogativa solamente dei polloi, dei molti, i quali non riescono a cogliere l'intima correlazione che vige tra questi due elementi.

Il secondo, vasto, contributo riguarda la gnoseologia secondo Empedocle di Agrigento (pp. 25-65). È interessante sottolineare come qui Montano affianchi al problema prettamente teoretico, l'altrettanto centrale elemento della ricostruzione storiografica, soprattutto secondo le testimonianze di Aristotele e di Sesto Empirico.

L'autore mette subito in risalto quelli che sono i limiti delle testimonianze aristoteliche e sestane del pensiero dei loro predecessori, mostrando come esse tendano a ricostruire il pensiero degli autori antichi secondo i parametri propri delle rispettive filosofie (ciò è particolarmente evidente nei casi in cui Aristotele passa in rassegna criticamente al pensiero di Empedocle per quanto riguarda i concetti di forma e causa). Montano sottolinea a più riprese il vizio di forma che sta alla base della ricostruzione della dottrina empedoclea da parte di Aristotele, il quale tende a sovrapporre i propri schemi concettuali alla dottrina dei predecessori. L'intento dell'autore in questo capito, al di là di ricostruire nella maniera più genuina possibile il senso originario della filosofia empedoclea, è quello di mettere in evidenza come il metodo storiografico sia un vero e proprio metodo filosofico: è infatti anche e soprattutto attraverso la storia della filosofia che una tesi filosofica può essere corroborata o indebolita.

Questa linea di pensiero portata avanti da Montano era già stata, invero, anticipata nel primo breve capitolo del libro intitolato Una proposta metodologica per lo studio di Parmenide (pp. 9-14), dove viene preso in esame il testo di A. Capizzi La porta di Parmenide (Roma 1975), in cui l'Eleate viene in un certo senso "spogliato" del suo ruolo di ontologista e scienziato a vantaggio di un'interpretazione in chiave strettamente politica della sua filosofia, mostrando quindi una forte tendenza ad attualizzare la figura di Parmenide; in risposta a ciò Montano suggerisce una ricostruzione onnicomprensiva, su base filologica, della figura storica dell'Eleate, che tenga conto non soltanto di un aspetto particolare della sua figura, ma che al contrario tenga insieme diverse attitudini del filosofo eleatico.

Di nuovo, la relazione tra sensi e ragione viene presa in esame anche per quanto concerne diversi aspetti di pensiero di Democrito in tre saggi che occupano la parte centrale del volume (Il metodo induttivo in Democrito? pp. 67-89; Il fenomeno e il discorso: il modello epistemologico in Democrito pp. 91-127; La genesi della credenza religiosa in Democrito pp. 129-150). Il discorso intorno a Democrito, seppur dilazionato in ben tre capitoli, viene affrontato da Montano con uno sguardo di insieme globale, mostrando come il rapporto tra sensi e ragione sia una delle parti più feconde della dottrina atomista. La domanda di esordio è la colonna portante dell'intera questione: «È possibile parlare di metodi di ricerca in autori pre - aristotelici?» (p. 67) È fuor di dubbio che il metodo scientifico trovi la sua sistematizzazione solo con Aristotele (questa è la tesi del Langerberg, riportata dall'autore), benché già con Ippocrate si erano avute prima approssimazioni di una precisa metodologia di ricerca. 
Il punto fondamentale da cui prende le mosse il ragionamento di Montano è, ancora una volta, l'impossibilità di affidarsi completamente a quelle che sono le testimonianze di altri autori, poiché ognuno di questi, alla domanda se la dottrina democritea abbia come elemento portante la conoscenza sensibile o la conoscenza razionale, tendono a privilegiare o l'uno o l'altro elemento (ciò a seconda di quella che è l'esigenza filosofica dello storiografo, in questo caso ancora Aristotele e Sesto Empirico), depotenziando quella che è l'idea centrale del pensiero dell'Abderita che vede senso e ragione muoversi di pari passo («La ragione può e deve giungere là dove il senso fallisce», pag. 77). Democrito dunque non esclude la sensibilità nella sua scala della conoscenza, bensì la pone come essenziale punto di partenza per conoscere il modo in cui la realtà si manifesta, ma nel momento in cui è necessario ricorrere ai principi, ecco subentrare la ragione (definita "misera" solo nella misura in cui essa tenti di operare da sé), tuttavia ciò non comporta, nel caso dell' Abderita, l'acquisizione di un metodo rigorosamente induttivo.

Anche nel secondo contributo viene ulteriormente sviluppata questa parte della teoria atomista: l'autore mostra come la teoria di Democrito debba essere letta alla luce di una sostanziale visione "co-operativa" di sensibilità e ragione. Riprendendo in parte quanto già scritto nel primo contributo sull'atomismo, Montano ci mostra come la teoria democritea presenti un dualismo (che si snoda in categorie fondamentali come quelle di "fenomeno" e "discorso") che è in realtà soltanto apparente, in quanto sensibilità ed intelletto hanno, a livello gnoseologico, funzioni complementari. Segue un'attenta analisi terminologica su alcuni concetti chiave della filosofia dell'Abderita, che viene riformulata da Aristotele e da Sesto E. sotto due differenti accezioni: sensismo secondo lo Stagirita e razionalismo per il secondo. La conclusione a cui perviene l'autore non esclude nessuno dei due elementi conoscitivi: «Gli elementi semplici, atomi e vuoto, infatti, rappresentano ambedue la struttura dei fenomeni e perciò non sono percepibili attraverso i sensi. Per coglierli allora c'è bisogno, allora, di far ricorso alla conoscenza genuina, che, andando oltre i dati percettivi, senza però negarli, ne mostra il reale fondamento» (pag. 124).

Questo ragionamento democriteo in merito all'utilizzo della ragione investe anche la communis opinio in merito alla credenza religiosa: secondo l'Abderita, la credenza religiosa sta in piedi su una sorta di totale sentimento di smarrimento dei "molti" di fronte a fenomeni complessi della natura: in virtù di ciò essi «foggiano l'idolo del caso» (p. 140), questo atteggiamento di impotenza di fronte al fenomeno genererebbe la credenza religiosa in forze soprannaturali; in risposta a ciò, Democrito osserva come la causa di questa credenza religiosa sia la povertà razionale dei "molti" a non considerare che ogni cosa che avviene in natura è razionalmente dimostrabile.

$\mathrm{Su}$ un binario diverso rispetto a queste tesi democritee sembra svilupparsi la figura di Gorgia. Sebbene la critica dell'Agrigentino sia rivolta, come è noto, essenzialmente contro l'eleatismo e le sue astrazioni logico - ontologiche, Montano pone invece qui l'accento su un aspetto particolare del pensiero del sofista, e cioè quello secondo cui non vi è corrispondenza tra pensiero ed essere (in pratica capovolgendo quanto affermato da Parmenide in DK 28 B 3; 8,34). Sulla base di ciò, il sofista scinde nettamente sensibilità e ragione, per cui il discorso non può più prendere l'iniziativa da sé, ma in qualche modo 
«essa [scil. La logica] è diventata tributaria dell'esperienza» (p. 167), nel senso che non può più da sola, intavolare ragionamenti che hanno pretesa di verità, prescindendo dal dato empirico. Tutto ciò è giustificato dal fatto che se le cose viste, udite o toccate provengono dall'esterno (perciò si manifestano in maniera evidente), allora il pensiero, essendo espressione di un'astrazione mentale proveniente dall'interiorità, non può avere corrispondenza con l'essere, ma deve necessariamente tirarsi indietro e sottostare a quella che è il dato dell'evidenza sensibile.

Un ulteriore e vivace capitolo riguarda l'analisi del metodo per ipotesi nel Menone. Montano qui analizza infatti come, accanto ai ben più noti metodi di indagine platonici, vale a dire quello giovanile «socratico» e quello dialettico, il metodo per ipotesi si affianchi a pieno titolo a questi ultimi come efficace mezzo per la ricerca e la scoperta del vero.

In particolare, il metodo ipotetico si fonda sulla teoria platonica dell'anamnesi, il che significa che «Solo ammettendo, infatti, che nell'animo dell'uomo ci siano parti, anche poche ed irrelate, di verità - scrive Montano - è possibile sconfiggere l'aporia eristica

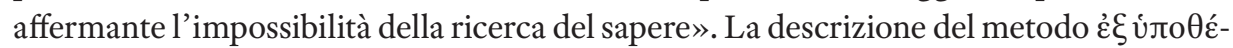
$\sigma \varepsilon \omega \varsigma$ e le sue applicazioni sono espresse al fine di dimostrare come la sua estensione, che racchiude in sé come conseguenza anche la teoria della reminescenza, si ponga non in contraddizione, ma in linea di continuità con il metodo giovanile socratico ed il metodo dialettico esposto nei dialoghi della maturità.

Infine, parallelamente ad alcuni capitoli dedicati a Plutarco (pp. 239-246), alla diatriba tra filosofi ed astrologi nel V secolo e sulla filosofia nella Magna Graecia (pp. 247-266), Montano si sofferma in un capitolo A difesa del classico (pp. 267-284) al fine di far emergere l'attualità non solo delle tematiche trattate ma anche, di converso, della riscoperta del testo classico, non necessariamente filosofico. Riprende forma allora quel tentativo di non slegare filosofia e storia della filosofia già richiamato nelle pagine precedenti. In particolar modo, l'ultimo capito di Methodos lascia trasparire una volontà dell'autore di attualizzare le problematiche inerenti lo studio dei classici. Dopo aver mostrato come, nella storia del pensiero occidentale, il ricorso al "classico" sia stata prerogativa dei più grandi filosofi ed umanisti, Montano passa ad analizzare il senso stesso di questo "ritorno al passato". L'autore mostra come il riferimento al classico non sia una mera attività contemplativa verso qualcosa che c'era e non c'è più, bensì esso si pone come elemento fecondo per risvegliare la coscienza del sé all'interno di un soggetto (singolare o collettivo) che corrobori la propria identità attraverso il raffronto con le auctoritates e comprenda quanto di quella specifica tradizione ancora sia presente in esso e quanto invece vi sia di differente, cosicché venga salvaguardata e rinvigorita l'identità di chi si confronta con la tradizione.

In conclusione, credo che i maggiori meriti del testo esaminato siano, oltre alla pregevole ricostruzione del pensiero dei vari autori affrontati, 1) quello di aver dimostrato l'attualità dei contenuti della filosofia antica, 2) quello di aver posto l'attenzione su quel nucleo comune tra filosofia e storia della filosofia che molto spesso si tende a scindere, ma che invece rappresentano due facce della stessa medaglia. 